CERN-PH-TH/2010-011

\title{
Leptons from Dark Matter Annihilation in Milky Way Subhalos
}

\author{
James M. Cline* ${ }^{1,2}$ Aaron C. Vincent ${ }^{\dagger},{ }^{2}$ and Wei Xue ${ }^{\ddagger 2}$ \\ ${ }^{1}$ Theory Division, CERN, CH-1211, Genève, Switzerland \\ ${ }^{2}$ Department of Physics, McGill University, 3600 Rue University, Montréal, Québec, Canada H3A 2T8
}

(Dated: October 25, 2018)

\begin{abstract}
Numerical simulations of dark matter collapse and structure formation show that in addition to a large halo surrounding the baryonic component of our galaxy, there also exists a significant number of subhalos that extend hundreds of kiloparsecs beyond the edge of the observable Milky Way. We find that for dark matter (DM) annihilation models, galactic subhalos can significantly modify the spectrum of electrons and positrons as measured at our galactic position. Using data from the recent Via Lactea II simulation we include the subhalo contribution of electrons and positrons as boundary source terms for simulations of high energy cosmic ray propagation with a modified version of the publicly available GALPROP code. Focusing on the DM DM $\rightarrow$ e annihilation channel, we show that including subhalos leads to a better fit to both the Fermi and PAMELA data. The best fit gives a dark matter particle mass of $1.2 \mathrm{TeV}$, for boost factors of $B_{\mathrm{MH}}=90$ in the main halo and $B_{\mathrm{SH}}=1950-3800$ in the subhalos (depending on assumptions about the background), in contrast to the $0.85 \mathrm{TeV}$ mass that gives the best fit in the main halo-only scenario. These fits suggest that at least a third of the observed electron cosmic rays from DM annihilation could come from subhalos, opening up the possibility of a relaxation of recent stringent constraints from inverse Compton gamma rays originating from the high-energy leptons.
\end{abstract}

\section{INTRODUCTION AND SUMMARY}

Recent observations of the spectrum of electrons and positrons by the Fermi collaboration [1] and of the positron fraction $e^{+} /\left(e^{+}+e^{-}\right)$by the PAMELA experiment [2] hint at a possible new source of cosmic ray $e^{+}$ and $e^{-}$in the TeV energy region. According to recent models [3-9], these excesses could be the signal of dark matter (DM) annihilation via a dark sector gauge boson that allows a Sommerfeld-type enhancement at low velocities. Best fits to the electron-positron spectra indicate that the dark matter candidate $\chi$ that annihilates within the galaxy should have a mass of around $M_{\mathrm{DM}} \simeq 1$ $\mathrm{TeV}$, and annihilate into two pairs of light leptons via the process DM DM $\rightarrow \phi \phi \rightarrow 4 e$ or DM DM $\rightarrow \phi \phi \rightarrow 4 \mu$. The particle $\phi$ should furthermore be light enough not to decay into $p \bar{p}$ pairs since excess antiprotons are not observed by PAMELA. ${ }^{1}$

While the visible galaxy spans a diameter of approximately $40 \mathrm{kpc}$ and a height of $8 \mathrm{kpc}, N$-body simulations $[10,11]$ predict a roughly spherical structure of dark matter subhalos whose peak concentration occurs $\sim 70$ $\mathrm{kpc}$ from the galactic center (GC) and extends as far as several thousand kpc. Relative velocities between particles in these regions are one to two orders of magnitude smaller than in the Milky Way's main halo, and the rela-

\footnotetext{
*jcline@hep.physics.mcgill.ca

†vincenta@hep.physics.mcgill.ca

${ }^{\ddagger}$ xuewei@hep.physics.mcgill.ca

1 however see ref. [7] for arguments that this constraint may not be necessary, due to astrophysical uncertainties in the background model
}

tive overdensity of such regions make them ideal sources of DM annihilation products. This has been explored by other authors in the context of gamma ray signals originating from subhalos $[8,12-16]$ and found to be significant. These gamma rays, which originate from finalstate radiation, are not the main product of this class of DM annihilation; rather they are by-products of charged particles and neutral pions.

In this work we consider the possibility that the excess leptons observed by PAMELA and Fermi/LAT themselves have a strong component originating in the subhalos. This possibility was previously considered in ref. [17], but there it was assumed that one or two nearby subhalos would dominate any additional contribution to the signal. Here we will show that the best fits to the data are found by taking into account the full ensemble of substructures. It will be seen that the subhalos that individually contribute weakly to the lepton flux are nevertheless so numerous that their combined effects cannot be neglected.

We used a modified version of the GALPROP cosmic ray propagation code, in which leptons from distant subhalos give a new source term at the boundary of the diffusion zone. The data of the Via Lactea II simulation [10] are taken as our model for the subhalos. We allow for independently adjustable boost factors for the main halo and subhalos, motivated by the fact that Sommerfeld enhancement of the annihilation cross section can be much greater in the subhalos due to their lower velocity dispersion [3]. If we also allow the background electron and positron flux normalizations to be rescaled, as in references $[9,18,19]$, we find that the inclusion of subhalos gives a much better fit to both cosmic ray data sets, with the best-fit DM particle mass of $M_{\mathrm{DM}}=1.2 \mathrm{TeV}$.

On the other hand, if the $e^{+}$and $e^{-}$backgrounds are 
instead fixed at the GALPROP output level, it is known that there is a discrepancy between the boost factors needed for explaining PAMELA and Fermi, even in the standard main halo-only scenario. This discrepancy remains in the subhalo scenario, where we find that a DM mass of $M_{\mathrm{DM}}=2.2 \mathrm{TeV}$ improves the fit to the Fermi data, whereas the fit to PAMELA is not improved.

Our results suggest that the inclusion of leptons from DM annihilation in the subhalos surrounding the Galaxy should affect not only the amplitude, but also the shape of the observed spectrum due to inverse Compton scattering (ICS) of the leptons with the radiation fields inside the observable galaxy. This is potentially important because the most recent constraints on this effect $[18,19]$ effectively rule out the DM annihilation interpretation of the Fermi excess, and leave only a very reduced corner of parameter space consistent with PAMELA. We hope to quantitatively address the question of whether substructure indeed allows for relaxation of these constraints in the near future.

In section 2 we briefly describe GALPROP and our choices of parameters for cosmic ray propagation. Section 3 details the modifications we made to GALPROP in order to include the $e^{+} e^{-}$pairs from DM annihilation in the subhalos. The results are presented in section 4 , and conclusions in section 5 .

\section{COSMIC RAY PROPAGATION MODELS}

Inside the diffusive zone of the Galaxy, cosmic ray species propagate according to the transport equation $[20]$

$$
\begin{aligned}
\frac{\partial \psi}{\partial t}= & q(r, z, p)+\nabla \cdot\left(D_{x x} \nabla \psi-\vec{V}_{c} \psi\right) \\
& +\frac{\partial}{\partial p} p^{2} D_{p p} \frac{\partial}{\partial p} \frac{1}{p^{2}} \psi-\frac{\partial}{\partial p}\left[\frac{d p}{d t} \psi-\frac{p}{3}\left(\nabla \cdot \vec{V}_{c}\right) \psi\right] .
\end{aligned}
$$

$\psi(\vec{x}, p, t)$ is the particle density per unit momentum $p \equiv|\vec{p}|, q(\vec{x}, p)$ is the source term, $D_{x x}$ is the energydependent diffusion coefficient, $D_{p p}$ quantifies reacceleration via diffusion in momentum space and $\vec{V}_{c}$ is the convection velocity. In the case of composite species, terms accounting for radiative decay and fragmentation must furthermore be included. For $D_{x x}$ we use the parametrization [21]

$$
D_{x x}=D_{0 x x}\left(\frac{E}{4 \mathrm{GeV}}\right)^{\delta}
$$

where $E$ is the particle energy and $D_{0 x x}$ is the diffusion coefficient at reference energy $E=4 \mathrm{GeV} .{ }^{2} D_{0 x x}$ and

\footnotetext{
2 More precisely, diffusion depends on particle rigidity, the energy divided by the charge. We assumed the particles have unit charge
}

the exponent $\delta$ are determined by fitting to heavy nuclei cosmic ray data.

There are two widely-used approaches to cosmic ray propagation within the galaxy. The first is a semianalytic model in which the baryonic component of the galaxy is accelerated in a thin disk at $z=0$ from which particles diffuse according to a Bessel series expansion until $z= \pm L_{\text {eff }}$, beyond which they freely escape. The second, fully numerical, approach implemented in the publicly available GALPROP [20] package uses a CrankNicholson scheme to solve eq. (1) within a diffusion zone of height $L_{\text {eff }}$ and radius $R_{\text {eff }}$. An advantage of the latter technique is that it allows the use of realistic maps of radiation and gas in the propagation scheme. While this is not the focus of our paper, it is relevant to point out that differences between models are responsible for differences between fits in recent dark matter annihilation models. This discrepancy has been known for some time; see for example the discussion in ref. [22]. Nevertheless, we shall henceforth focus exclusively on the numerical approach. Our simulations were run using a modified version of GALPROP 50.1p that was graciously provided by the authors of ref. [6] and which we further modified to handle subhalo sources.

The strongest available constraints on cosmic ray propagation models are ratios of secondary-to-primary species such as B/C or sub-Fe/Fe. The authors of ref. [21] conducted an exhaustive search of the GALPROP parameter space for input values that gave best fits to 12 secondary/primary cosmic ray experiments. For our simulation runs we took their best fit parameters: $D_{0 x x}=$ $6.04 \times 10^{28} \mathrm{~cm}^{2} \mathrm{~s}^{-1}\left(0.19 \mathrm{kpc}^{2} / \mathrm{Myr}\right), L_{\text {eff }}=5.0 \mathrm{kpc}$, $\delta=0.41$, with no convection. We used an Alfvén speed $V_{A}=31 \mathrm{~km} \mathrm{~s}^{-1}$, which gave a slightly better fit to the HEAO B/C data [23]. It should be noted that these parameters are quite different from the corresponding best fits of the semi-analytic model used, for example by Meade et al. [9].

\section{INCLUDING SUBHALO FLUX IN GALPROP}

The many-body simulation Via Lactea II [10], which modeled the evolution and collapse of more than $10^{9}$ particles over the history of a Milky Way-sized structure, resolves over 20,000 dark matter subhalos around the galactic host halo. The data characterizing each of these subhalos is publicly available [24]. While the visible galaxy is only some $40 \mathrm{kpc}$ across, these subhalos extend as far out as $4000 \mathrm{kpc}$ from the galactic center. Each subhalo is locally much denser than the host halo and has its own radial velocity dispersion profile. The annihilation rate of dark matter, proportional to $\rho^{2}$, should thus spike within these subsystems when compared to

here. 
the annihilation rate of diffuse DM particles of the host halo.

For a given subhalo $i$ at a distance $\ell_{i}$ from the edge of the diffusion zone of the galaxy, the flux of $e^{+}$or $e^{-}$on this boundary takes the form

$$
\frac{d \Phi_{i}}{d E}=B_{\mathrm{SH}}\langle\sigma v\rangle \frac{d N}{d E}\left(\ell_{i}\right) \int_{0}^{\infty} \frac{r^{2} \rho_{i}^{2}}{\ell_{i}^{2} M_{\mathrm{DM}}^{2}} d r
$$

where $B_{\mathrm{SH}}$ is an average boost factor for the subhalos due to Sommerfeld enhancement for example, and $\rho_{i}(r)$ is the mass density profile of the subhalo. The unboosted cross section is assumed to be $\langle\sigma v\rangle=3 \times 10^{-26} \mathrm{~cm}^{3} \mathrm{~s}^{-1}$ in accordance with the standard assumption that the DM abundance was determined by freeze-out starting from a thermal density. In a more exact treatment, the boost factor would be velocity dependent $[25,26]$ and appear within the average over DM velocities indicated by the brackets in $\langle\sigma v\rangle$. Moreover each subhalo in general has a different boost factor since the velocity dispersions that determine $B_{\mathrm{SH}}$ depend on the size of the subhalo [13]. For this preliminary study, we simply parametrize the effect by an average boost factor, where the averaging includes the sum over all subhalos as well as the integration over velocities.

The energy spectrum $d N / d E$ of electrons from the DM annihilations is taken for simplicity to be a step function at the interaction point, $d N / d E=M_{\mathrm{DM}}^{-1} \Theta\left(M_{\mathrm{DM}}-E_{0}\right)$, where $E_{0}$ is the energy immediately following the annihilation. We are interested in models where the DM particles initially annihilate into two hidden sector gauge or Higgs bosons, each of which subsequently decays into $e^{+} e^{-}$[3]. The four-body phase space would thus be a more exact expression for $d N / d E$, but the step function has the correct qualitative shape and is simpler to implement in GALPROP.

The energy of the electron at the edge of the galaxy is reduced from its initial value $E_{0}$ by scattering with $\mathrm{CMB}$ photons before reaching the galaxy (starlight, infrared radiation and synchrotron radiation are only important in the inner galaxy [27]), according to the loss equation $d E / d \ell=-\kappa E^{2}[9]$ where $\kappa=\left(4 \sigma_{T} / 3 m_{e}^{2}\right) u_{\mathrm{CMB}}=6.31 \times$ $10^{-7} \mathrm{kpc} \mathrm{GeV}^{-1}, \sigma_{T}=\frac{8 \pi}{3}\left(\alpha_{E M} \hbar / m_{e} c\right)^{2}$ is the Thomson cross-section and $u_{\mathrm{CMB}}=0.062 \mathrm{eV} / \mathrm{cm}^{3}$ is the present energy density of the CMB. It is convenient to write the solution in the inverted form: $E_{0}=(-\kappa \ell+1 / E(\ell))^{-1}$ for substitution into $d N / d E$. Numerically, we find that the losses outside the diffusion zone make a small correction, and that the distinction between $E_{0}$ and $E(\ell)$ is not important here.

Each subhalo is characterized by a density profile that has been fit to the Einasto form

$$
\rho_{i}=\rho_{s, i} \exp \left[-\frac{2}{\alpha}\left(\left(\frac{r}{r_{s, i}}\right)^{\alpha}-1\right)\right]
$$

with $\alpha=0.17$ [15]. The scale radius is found to be proportional to the radius $r_{v_{\max }}$ at which the veloc-

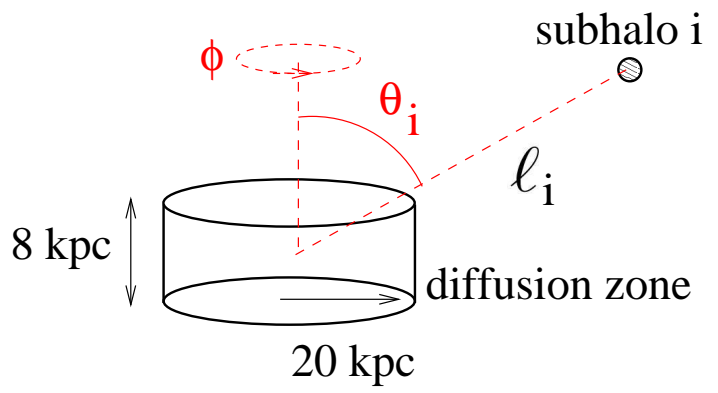

FIG. 1: Geometry of a subhalo shining leptons on the boundary of diffusion zone of the galaxy.

ity dispersion is at a maximum, through the relation $r_{s} \cong r_{v_{\max }} / 2.212$, while the prefactor scales with the maximum velocity $v_{\max }$ as $\rho_{s} \cong v_{\max }^{2} /\left(0.897 \cdot 4 \pi r_{s}^{2} G\right)$.

To incorporate the contribution (3) to the lepton flux from the subhalos in GALPROP, we add delta function source terms to $q(r, z, p)$ for the cylindrical surface bounding the diffusion zone, as illustrated in fig. 1:

$$
\begin{aligned}
q_{\text {disk }} & =2 \delta(z \pm h / 2) \sum_{i} \frac{d \Phi_{i}}{d E} \cos \theta_{i} \\
q_{\text {band }} & =2 \delta(r-R) \sum_{i} \frac{d \Phi_{i}}{d E} \sin \theta_{i}
\end{aligned}
$$

where $h$ and $R$ are respectively the height and radius of the cylinder. The factor of 2 corrects for the fact that sources in GALPROP have no directionality, whereas the flux impinging on the surface is inward. The sum is over the 20,048 resolved subhalos in the Via Lactea II simulation. In addition, the sources were averaged over the azimuthal angle $\phi$ because GALPROP assumes cylindrical symmetry in its 2D mode (and the 3D mode runs too slowly for our purposes). Finally, the distance $\ell_{i}$ must be corrected for subhalos that are close to the diffusion zone; rather than the distance to the center of the galaxy, it should be the distance to the cylindrical boundary. On average, this is a reduction by $17 \mathrm{kpc}$ compared to the distance to the galactic center.

Although most subhalos were located outside of the diffusion zone, there are 143 lying inside, whose contribution required special treatment. Assuming approximate isotropy, we took their entire flux of $e^{+}+e^{-}$to be pumped into the diffusion zone from the boundary rather than from their individual positions. Treating them in this manner allowed us to group their contribution with that of the other subhalos and thus consider a single average boost factor for all subhalos. This approximation would break down if one subhalo happened to be very close to our position in the galaxy, but treating such a case would anyway require going beyond the standard cylindrical symmetry (2D) mode of GALPROP and using the much slower 3D mode. We believe this treatment is conservative in the sense that it should only underestimate the contributions of the nearby subhalos. 


\section{NUMERICAL RESULTS}

We compared the observed flux of positrons and electrons generated by dark matter annihilation within the main halo $(\mathrm{MH})$ to a scenario in which both main halo and subhalo $(\mathrm{MH}+\mathrm{SH}) \mathrm{DM}$ annihilation occurs. We restricted our analysis to the DM DM $\rightarrow$ 4e channel. This channel is simpler to analyze, and is somewhat less constrained by inverse Compton gamma ray constraints than the other 4-lepton final-state models, or those with only two leptons [9].

For the $\mathrm{MH}$ only scenario, the annihilation crosssection was augmented by a constant boost factor $B_{\mathrm{MH}}$, representing the effect of Sommerfeld or some other kind of enhancement [3, 28-30]. This was varied in order to find a best fit to each data set. A similar approach was used in the case of $\mathrm{MH}+\mathrm{SH}$, where we varied the $\mathrm{MH}$ and $\mathrm{SH}$ boost factors independently. This is justified by the expectation that Sommerfeld enhancement should be significantly larger in the subhalos due to their lower velocity dispersions [3]. The subhalo boost factor $B_{\mathrm{SH}}$ might also have further contributions besides Sommerfeld enhancement, such as the presence of unresolved subhalos that we do not take into account [15], as well as substructure within the subhalos themselves [13].

We minimized the chi squared coefficient

$$
\chi^{2}=\sum_{i} \frac{\left(\xi_{i, \exp }-\xi_{i, \text { model }}\right)^{2}}{\sigma_{i, \exp }^{2}}
$$

where the sum runs over the measured or predicted values of $\xi$, which stands for either $E^{3} d\left(\psi_{e^{+}}+\psi_{e^{-}}\right) / d E$ in the case of the 25 Fermi data points, or $\psi_{e^{+}} /\left(\psi_{e^{+}}+\psi_{e^{-}}\right)$ in the case of the PAMELA data, and $\psi_{e^{ \pm}}$is the flux of electrons or positrons. When fitting to PAMELA we excluded the first 8 of 16 data points, following the usual assumption that the dip relative to the background is accounted for by solar modulation effects [7].

\subsection{Freely-varying background}

Our best fits to the PAMELA and Fermi data were obtained by letting the astrophysical background electrons and positrons be rescaled by overall normalization factors, which was also the approach taken in references [9, 18, 19]. Adding subhalo contributions significantly improved the fits to both the Fermi and the PAMELA data. While the best overall fit with only $\mathrm{MH}$ electrons was for $M_{\mathrm{DM}}=850 \mathrm{GeV}\left(\chi_{\text {total }}^{2}=34.3\right)$, the MH+SH scenario gave a best fit at $M_{\mathrm{DM}}=1.2 \mathrm{TeV}\left(\chi_{\text {total }}^{2}=16.5\right)$. In this case $31 \%$ of the DM electron + positron flux at the sun's location originated from subhalos.

A summary of these results is presented in the top portion of Table I. The predictions for $\mathrm{MH}$ and $\mathrm{MH}+\mathrm{SH}$ scenarios are shown for the total $e^{+}+e^{-}$flux in figures 2 and 3 and for the positron fraction in figures 4 and 5 . The value of $\chi^{2}$ versus $M_{\mathrm{DM}}$ is shown in fig. 6 , marginalizing

\begin{tabular}{|l|l|l|l|l|l|c|}
\hline \multicolumn{7}{|c|}{ Freely-varying background } \\
\hline & $M_{\mathrm{DM}}$ & $\chi_{\text {Fermi }}^{2}$ & $\chi_{\text {PAMELA }}^{2}$ & $\chi_{\text {total }}^{2}$ & $B_{\mathrm{MH}}$ & $B_{\mathrm{SH}}$ \\
\hline $\mathrm{MH}$ & $0.85 \mathrm{TeV}$ & 15.5 & 18.7 & 34.3 & 90.3 & - \\
$\mathrm{MH}+\mathrm{SH}$ & $1.2 \mathrm{TeV}$ & 2.3 & 14.2 & 16.5 & 92.8 & 3774 \\
\hline \multicolumn{7}{|c|}{ Fixed GALPROP background } \\
\hline $\mathrm{MH}$ & $1.0 \mathrm{TeV}$ & 8.2 & 144 & 152.2 & 110 & - \\
$\mathrm{MH}+\mathrm{SH}$ & $2.2 \mathrm{TeV}$ & 2.1 & 175 & 177.1 & 146 & 1946 \\
\hline
\end{tabular}

TABLE I: Best fit scenarios. Top: when the background positron and electron spectra were allowed to vary by an overall factor; this corresponds to the best overall fit to the data. Bottom: using background that was fixed at GALPROP's normalization. In this case we used the best fit to Fermi, since the best overall fit gave values of electron + positron flux that were ruled out by the Fermi data. MH: main halo DM annihilation only. $\mathrm{MH}+\mathrm{SH}$ : subhalo annihilation included. $m_{D M}$ is the DM mass that gives the best fit and $\chi_{i}^{2}$ are the chi squared fits to the respective experiments as described in eq. (6). $B_{\mathrm{MH}}$ and $B_{\mathrm{SH}}$ are the boost factors necessary for $\mathrm{MH}$ and $\mathrm{SH}$ annihilation cross sections, respectively. Note that the addition of a subhalo contribution greatly improves the best fit for both Fermi and PAMELA. The required DM mass is larger because of the energy loss suffered by electrons propagating to us from the galactic edge.

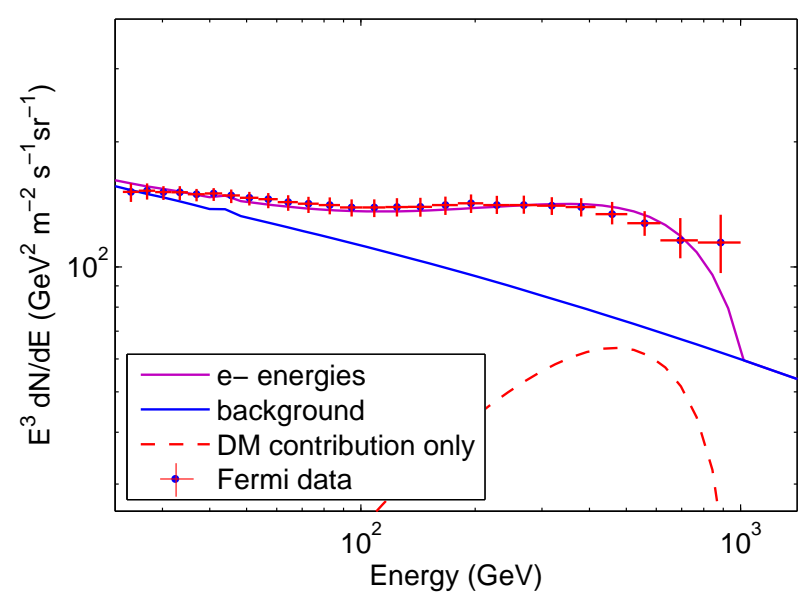

FIG. 2: Fermi data and prediction for $e^{+}+e^{-}$flux of the best main-halo-only fit to Fermi and PAMELA data, with an unconstrained background.

over the background normalizations. For the minimum $\chi^{2}$ point of the $\mathrm{MH}+\mathrm{SH}$ model, the background electrons had to be reduced to $97 \%$ of their predicted values, while background positrons were rescaled to $137 \%$ of the GALPROP output.

The optimal boost factors of 90 for the main halo and 3800 for the subhalos are quite reasonable from the point of view of DM models that give Sommerfeld-enhanced cross sections [3]. We leave for future work the issue of detailed particle physics model building to match these and other features of the best-fitting models. 


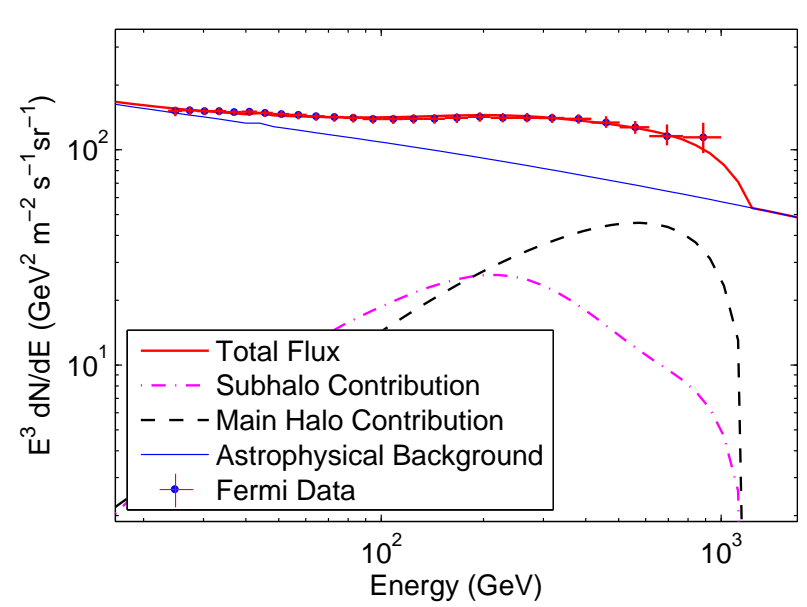

FIG. 3: Same as fig. 2, but now including subhalo contributions to the lepton fluxes.

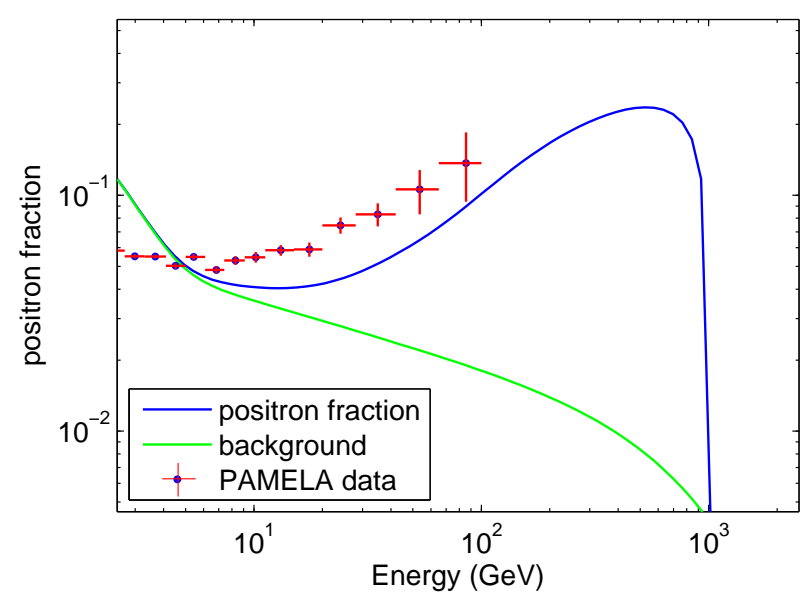

FIG. 4: PAMELA data and predicted positron fraction of the best main-halo-only fit to Fermi and PAMELA data, with an unconstrained background.

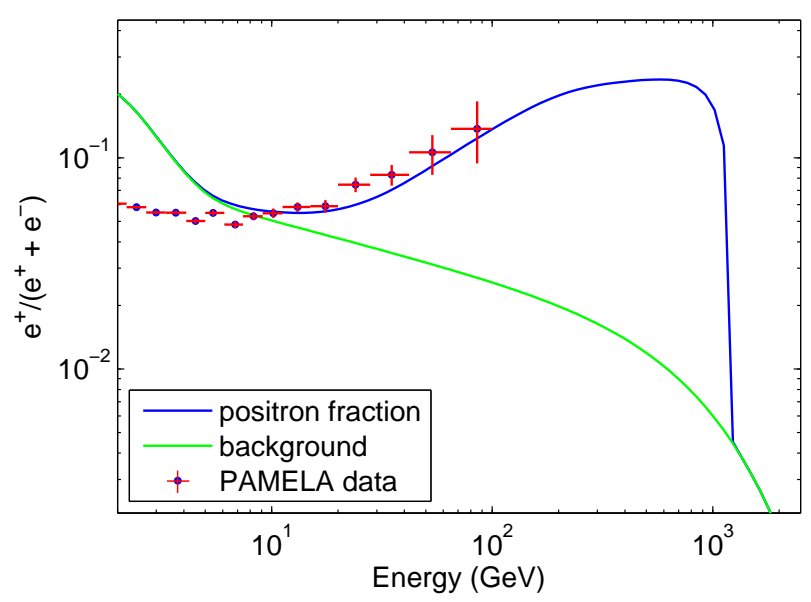

FIG. 5: Same as fig. 4, but now including subhalo contributions to the lepton fluxes.

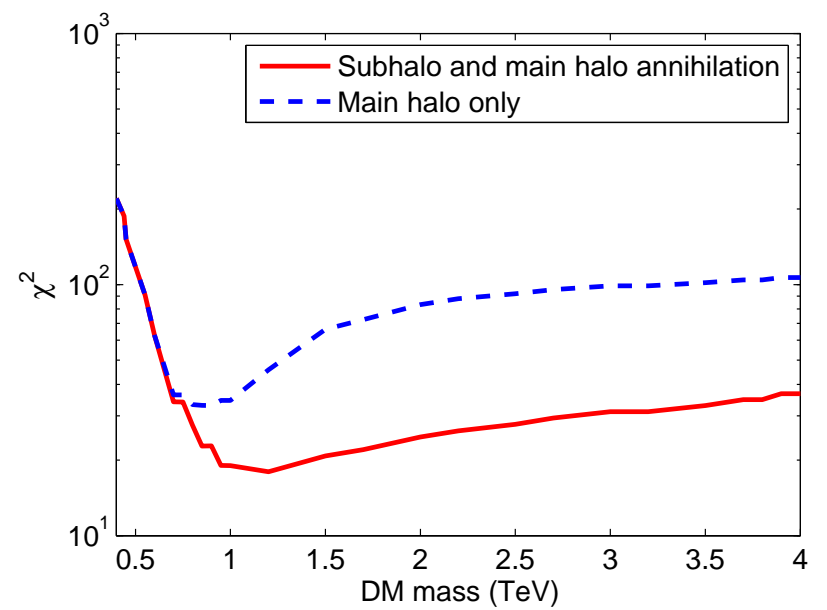

FIG. 6: Combined $\chi^{2}$ for the Fermi and PAMELA data as a function of the dark matter mass, for the unconstrained background. Dashed (blue) line: main halo DM annihilation only. Solid (red) line: subhalo and main halo contributions combined.

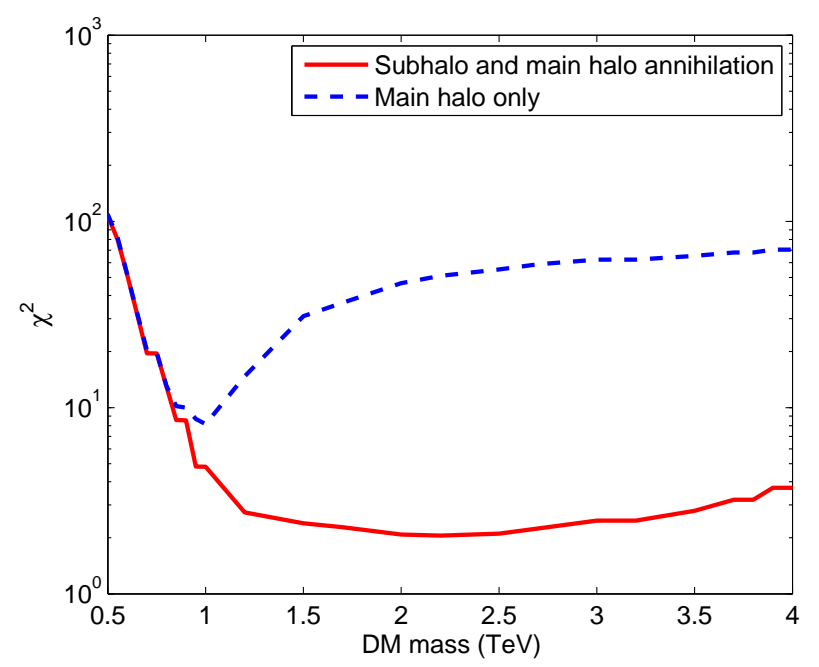

FIG. 7: $\chi^{2}$ versus $M_{\mathrm{DM}}$ for the Fermi $e^{+}+e^{-}$data using the GALPROP constrained background. Dashed (blue) line: main halo only. Solid (red) line: subhalos plus main halo.

\subsection{Constrained background}

We performed a second analysis by taking the electron and positron backgrounds to be those predicted by GALPROP. In this case, although there is no good simultaneous fit to the combined PAMELA and Fermi data, we nevertheless find that $\mathrm{SH}$ contributions improve the fit. In rough agreement with ref. [6], we find that the PAMELA data require a boost factor several times higher than that needed to fit the Fermi data.

The plots of $\chi^{2}$ versus $M_{\mathrm{DM}}$, for both the MH-only and $\mathrm{MH}+\mathrm{SH}$ models, are shown respectively for the Fermi and PAMELA data in figures 7 and 8 . It is striking that 


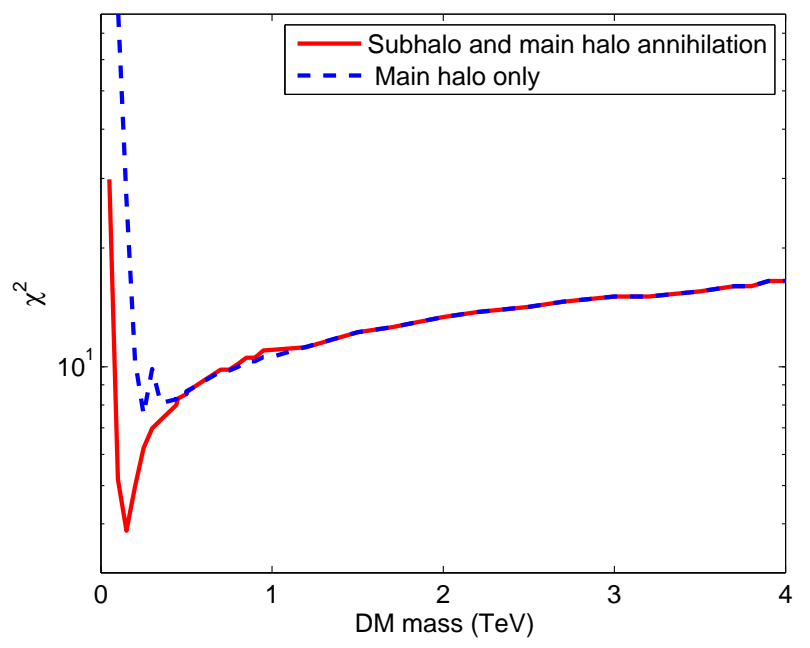

FIG. 8: $\chi^{2}$ versus $M_{\mathrm{DM}}$ for the Pamela positron fraction data, using the GALPROP constrained background. Dashed (blue) line: main halo only. Solid (red) line: subhalos plus main halo.

the best-fit DM mass becomes significantly larger and less constrained in the fit to the Fermi data including subhalos, fig. 8. The increase in the required DM mass is due to the energy lost by electrons and positrons during propagation from the edge of the diffusion zone to our position. The best fit to the Fermi data has $M_{\mathrm{DM}}=2.2$ $\mathrm{TeV}\left(\chi^{2}=2.05\right)$ with $\mathrm{SH}+\mathrm{MH}$, compared with $M_{\mathrm{DM}}=1$ $\mathrm{TeV}\left(\chi^{2}=8.15\right)$ in the $\mathrm{MH}$ only case. The required boost factors for these fits are $B_{\mathrm{MH}}=146$ and $B_{\mathrm{SH}}=1946$ for $\mathrm{SH}+\mathrm{MH}$, in contrast with $B_{\mathrm{MH}}=110$ for $\mathrm{MH}$ only. The best fit cases for the $e^{+}+e^{-}$spectrum are shown in Figure 9 and the results are summarized in the bottom part of Table I. The corresponding positron fraction in each of these scenarios is shown in Figure 10.

The fit to PAMELA is also improved by the addition of SH positrons, but only at low DM mass, $M_{\mathrm{DM}}<500$ $\mathrm{GeV}$. However the best fit parameters for the PAMELA data by themselves lead to a prediction of the $e^{+}+e^{-}$ $E^{3} d N / d E$ spectrum that exceeds the Fermi data by more than $3 \sigma$, resulting in a $\chi^{2}=460$ fit to Fermi. The badness of this fit is evident in fig. 11.

\subsection{Relative contributions of subhalos}

It is interesting to quantify how much of the signal can be contributed by the subhalos relative to that coming from the main halo. We show the fraction of $e^{+}+e^{-}$pairs due to the subhalos, as a function of the DM mass, in fig. 12. For the best-fit values of the mass, this fraction is around $30 \%$, but for larger values of $M_{\mathrm{DM}}$ (yet still giving reasonable fits) it rises to $60 \%$ or more. This may be helpful for weakening the constraints on the model from production of gamma rays by inverse Compton scattering $[18,19]$. We hope to investigate this issue in the near

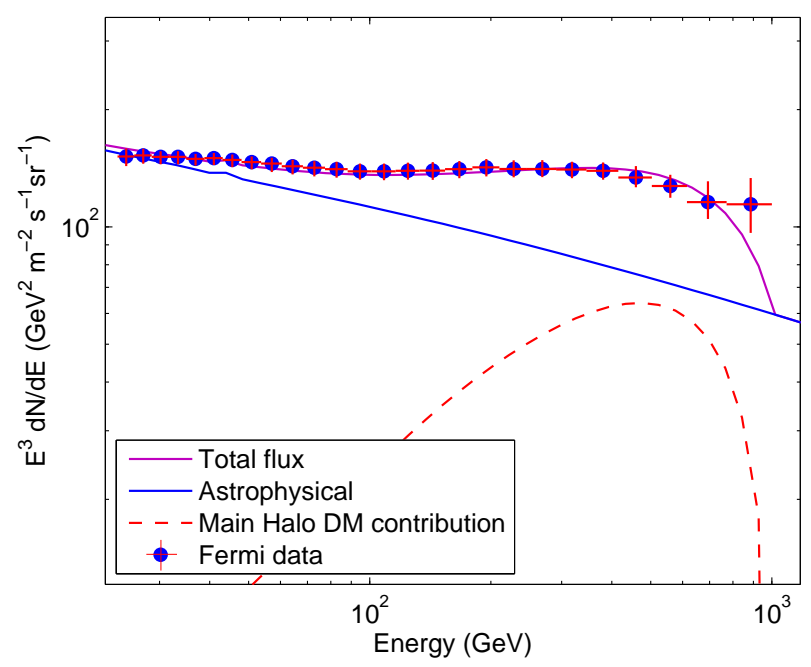

(a)

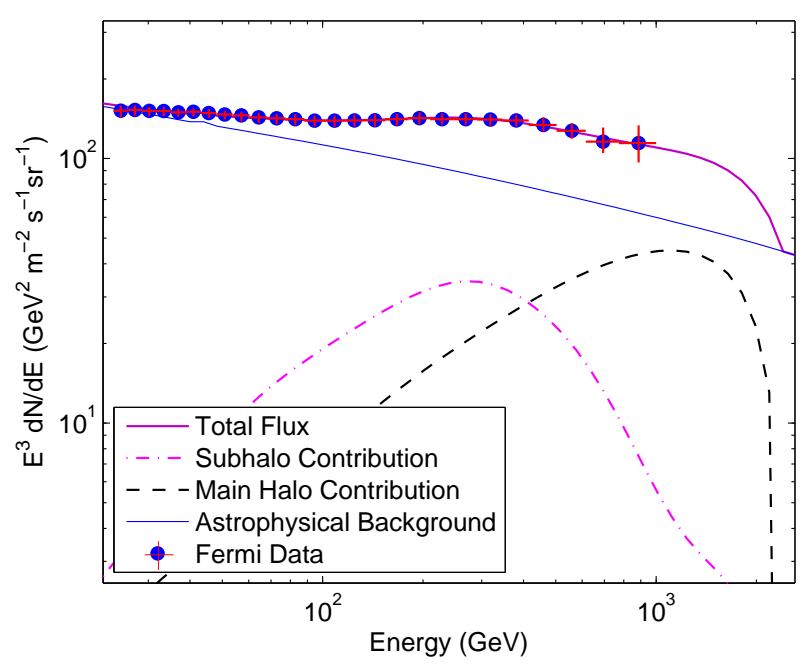

(b)

FIG. 9: Best fits to Fermi data. a) Main halo only, $M_{\mathrm{DM}}=1$ TeV, with a boost factor $B_{\mathrm{MH}}=110$. b) Subhalos plus main halo, $M_{\mathrm{DM}}=2.2 \mathrm{TeV}$ and $B_{\mathrm{MH}}=146, B_{\mathrm{SH}}=4825$.

future.

Another relevant issue is the hierarchy of contributions of subhalos relative to each other. One would like to know whether it was really necessary to add the contributions of all 20,000 subhalos, or if perhaps only the few closest ones dominate. Fig. 13 shows the distribution of subhalos contributing a given flux $\Phi$ (normalized to the contribution of the subhalo that gives the largest value $\left.\Phi_{\max }\right)$, weighted by the flux, and also the integral of this quantity. From the integral, we see that $50 \%$ of the total signal comes from subhalos whose individual intensities are less than $5 \%$ of the strongest one. Thus to get a quantitatively accurate estimate, it is necessary to include the very numerous subhalos whose intensity is low. This also 


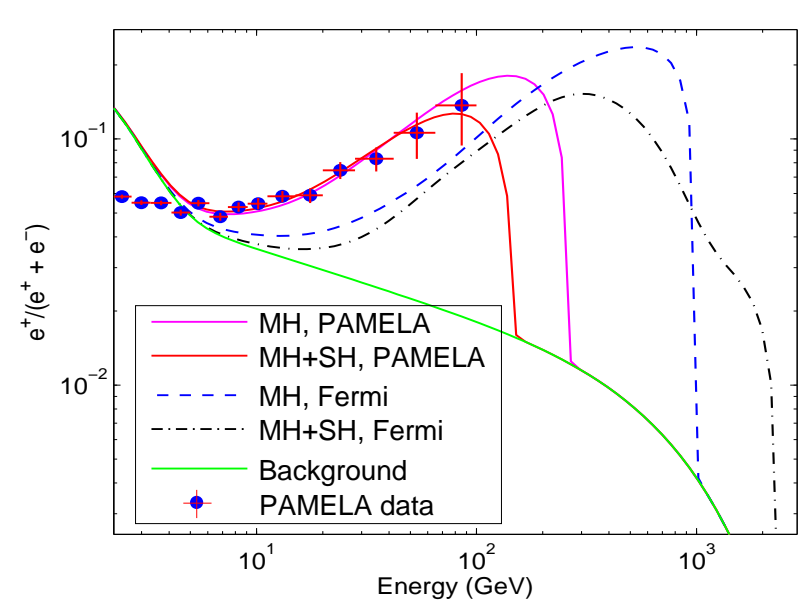

FIG. 10: Positron fraction for four of the best fit scenarios with constrained backgrounds. Top (solid) lines correspond to fits to PAMELA data only. Uppermost (magenta): $\mathrm{MH}$ only; $m_{D M}=250 \mathrm{GeV}, S_{M H}=225$. Lower (red): $\mathrm{MH}+\mathrm{SH}$; $m_{D M}=150 \mathrm{GeV}, S_{M H}=9.3, S_{S H}=509$. Bottom (dashed) lines correspond to the best fits of these scenarios to the Fermi data. Upper dashed (blue): $\mathrm{MH}$ only; $m_{D M}=1 \mathrm{TeV}, S_{M H}$ $=110$. Lower dot-dashed (black): $\mathrm{MH}+\mathrm{SH} ; m_{D M}=2.2 \mathrm{TeV}$, $S_{M H}=146, S_{S H}=4825$. Although the former set provide a better $\chi^{2}$, they predict a total $e^{+}+e^{-}$flux that conflicts with the Fermi data by at least $3 \sigma$ (see figure 11).

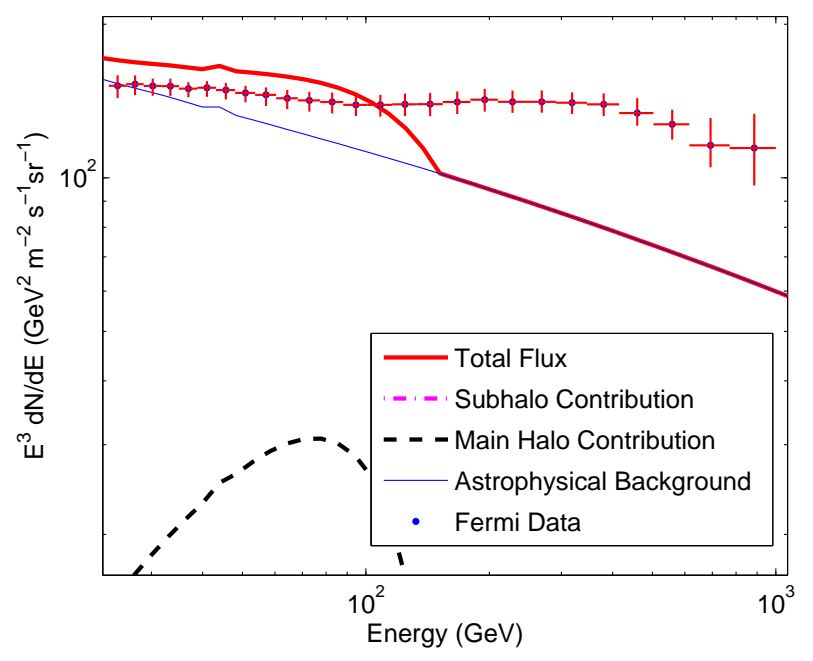

FIG. 11: $\mathrm{e}^{+}+\mathrm{e}^{-}$curve for best fit to PAMELA data only. $m_{D M}=150 \mathrm{GeV}, S_{M H}=9.3, S_{S H}=509$. Although the high energy tail could be compensated by other sources (e.g. pulsars), the fact that the model exceeds the data points at low energy leads us to disfavor this model. Note that the subhalo contribution is too small to be seen in this figure.

suggests that our computation is an underestimate, since we do not count the subhalos that are not resolved by the Via Lactea II simulation.

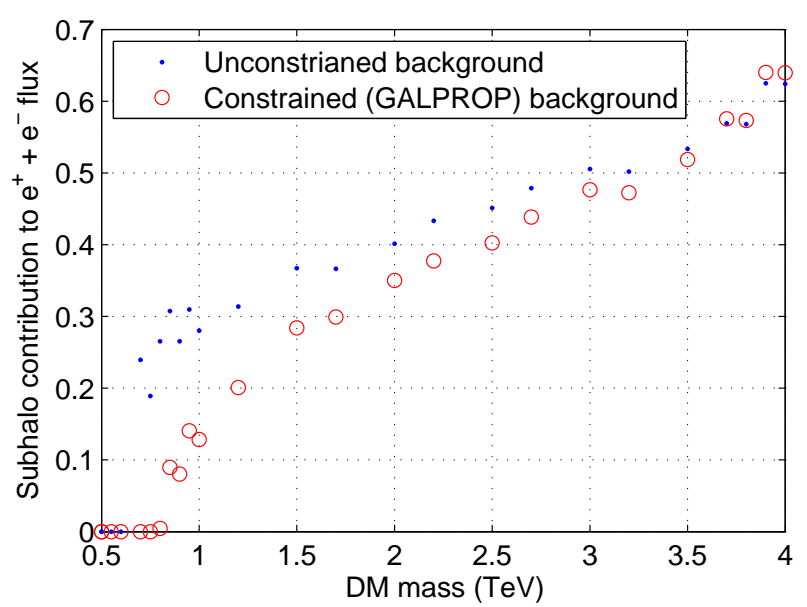

FIG. 12: Proportion of the total flux of $e^{+}+e^{-}$originating from subhalos as opposed to the main halo, as observed 8.5 $\mathrm{kpc}$ from the galactic center (the position of the solar system) in order to obtain a best fit to the Fermi data. Each point represents an individual simulation.

\section{CONCLUSIONS}

We have shown that the inclusion of electrons and positrons from the galactic subhalos can significantly alter the predictions from annihilating dark matter models. Using the Via Lactea II simulated data of the subhalo distribution around a Milky Way-like galaxy, we found that the contributions from substructure can give improved fits to the PAMELA and Fermi excess lepton data, and increase the value of the expected mass of the dark matter particle. A strong Sommerfeld boost coming from the low velocity dispersions of the subhalos, as well as

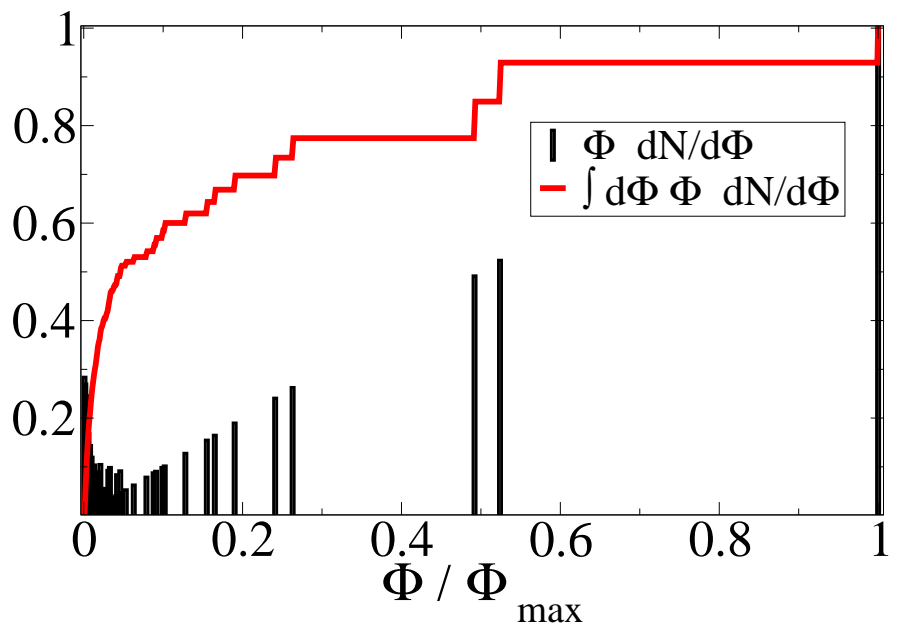

FIG. 13: Vertical bars (black): $\Phi d N / \mathrm{d} \Phi$, the distribution of subhalos contributing a given flux at the edge of the difusion zone, weighted by the flux. Continuous curve (red): integral of $\Phi d N / \mathrm{d} \Phi$. 
the uncounted contributions of subhalos unresolved by the Via Lactea II simulations, are possible sources for the boost factor necessary to obtain best our best fits to the data. According to these fits a third or more of the electron cosmic rays from DM annihilation could come from subhalos outside of the visible Milky Way.

The next step for future work will be to see whether the reduction of the flux from the main halo can weaken Fermi constraints on annihilating DM models due to the inverse Compton gamma rays produced by the highenergy leptons $[18,19]$. These constraints are sufficiently strong to rule out the DM interpretation of the Fermi lepton excess, under the usual assumption that all the $e^{+} e^{-}$ pairs are produced in the main halo. The constraints are strongest from data near the galactic center. By shifting the production away from the center to the subhalos, the constraints should be weakened, but whether the effect is large enough to reinstate the DM interpretation of the Fermi lepton observations is a quantitative question. In addition, one should satisfy other protohalo constraints
[31], extragalactic gamma background [32] and last scattering surface CMB constraints [33].

If it is possible for the scenario to pass these tests, it will be interesting to check whether specific particle physics models are able to give the average boost factors that we have treated as free parameters in this preliminary study.

As we were completing this work, ref. [34] appeared, which presents an analytical method for taking into account the effect of substructure on dark matter annihilation.

\section{Acknowledgments}

We thank Ilias Cholis, Ran Lu, Scott Watson and Neal Weiner for helpful discussions and correspondence concerning GALPROP. Our research is supported by NSERC (Canada) and FQRNT (Québec).
[1] The Fermi LAT, A. A. Abdo et al., Phys. Rev. Lett. 102, 181101 (2009), 0905.0025.

[2] PAMELA, O. Adriani et al., Nature 458, 607 (2009), 0810.4995 .

[3] N. Arkani-Hamed, D. P. Finkbeiner, T. R. Slatyer, and N. Weiner, Phys. Rev. D79, 015014 (2009), 0810.0713.

[4] F. Chen, J. M. Cline, and A. R. Frey, (2009), 0907.4746.

[5] I. Cholis, G. Dobler, D. P. Finkbeiner, L. Goodenough, and N. Weiner, (2008), 0811.3641.

[6] I. Cholis, D. P. Finkbeiner, L. Goodenough, and N. Weiner, (2008), 0810.5344.

[7] G. Kane, R. Lu, and S. Watson, (2009), 0906.4765.

[8] M. Kuhlen, J. Diemand, and P. Madau, (2008), 0805.4416.

[9] P. Meade, M. Papucci, A. Strumia, and T. Volansky, (2009), 0905.0480.

[10] J. Diemand et al., Nature 454, 735 (2008), 0805.1244.

[11] V. Springel et al., Mon. Not. Roy. Astron. Soc. 391, 1685 (2008), 0809.0898.

[12] S. Ando, Phys. Rev. D80, 023520 (2009), 0903.4685.

[13] J. Bovy, (2009), 0903.0413.

[14] M. D. Kistler and J. M. Siegal-Gaskins, (2009), 0909.0519.

[15] M. Kuhlen, P. Madau, and J. Silk, (2009), 0907.0005.

[16] M. Kuhlen, (2009), 0906.1822.

[17] P. Brun, T. Delahaye, J. Diemand, S. Profumo, and P. Salati, Phys. Rev. D80, 035023 (2009), 0904.0812.

[18] M. Cirelli, P. Panci, and P. D. Serpico, (2009), 0912.0663.

[19] M. Papucci and A. Strumia, (2009), 0912.0742.
[20] A. W. Strong and I. V. Moskalenko, Astrophys. J. 509, 212 (1998), astro-ph/9807150.

[21] M. Simet and D. Hooper, JCAP 0908, 003 (2009), 0904.2398.

[22] D. Maurin, R. Taillet, and F. Donato, Astron. Astrophys. 394, 1039 (2002), astro-ph/0206286.

[23] J. J. Engelmann, P. Ferrando, A. Soutoul, P. Goret, and E. Juliusson, Astron. Astrophys. 233, 96 (1990).

[24] http://www.ucolick.org/ diemand/vl/data.html.

[25] J. D. March-Russell and S. M. West, Phys. Lett. B676, 133 (2009), 0812.0559.

[26] M. Lattanzi and J. I. Silk, Phys. Rev. D79, 083523 (2009), 0812.0360.

[27] T. A. Porter and A. W. Strong, (2005), astro$\mathrm{ph} / 0507119$.

[28] J. Hisano, S. Matsumoto, M. M. Nojiri, and O. Saito, Phys. Rev. D71, 063528 (2005), hep-ph/0412403.

[29] M. Cirelli, A. Strumia, and M. Tamburini, Nucl. Phys. B787, 152 (2007), 0706.4071.

[30] M. Pospelov and A. Ritz, Phys. Lett. B671, 391 (2009), 0810.1502

[31] F.-Y. Cyr-Racine, S. Profumo, and K. Sigurdson, (2009), 0904.3933.

[32] G. Huetsi, A. Hektor, and M. Raidal, (2009), 0906.4550.

[33] T. R. Slatyer, N. Padmanabhan, and D. P. Finkbeiner, Phys. Rev. D80, 043526 (2009), 0906.1197.

[34] M. Kamionkowski, S. M. Koushiappas, and M. Kuhlen, (2010), 1001.3144. 